\title{
Favoritism of quantum dot inter-Coulombic decay over direct and multi-photon ionization by laser strength and focus
}

\author{
Anika Haller ${ }^{1,2}$ and Annika Bande ${ }^{1, a)}$ \\ 1) Institute of Methods for Material Development, Helmholtz-Zentrum Berlin \\ für Materialien und Energie GmbH, Albert-Einstein-Str. 15, 12489 Berlin, \\ Germany \\ 2) Institut für Chemie und Biochemie, Freie Universität Berlin, Takustr. 3, \\ 14195 Berlin, Germany
}

(Dated: 30 May 2018)

We study the dynamics of a two-electron system undergoing resonant excitation and inter-Coulombic decay (ICD) in a pair of quantum dots. The influence of the focus of the exciting laser on the ICD process is investigated for a $\pi$-pulse with a close look on competing processes, i.e. direct ionization and multi-photon excitations. We scan through the field strength up to six Rabi cycles to show that ICD is still verifiable after several population inversions. With novel analyses we determine for the first time populations of the different continuum states and thus conclude on the importance of several multi-photon excitation channels. Finally, we look into the influence of complex absorbing potentials on the dynamics.

a)Electronic mail: annika.bande@helmholtz-berlin.de 


\section{INTRODUCTION}

A persistently high interest exists in making electronic devices as small and efficient as possible while having in mind to operate fast and precise at the same time. As a result, the last decades of research brought forth revolutionary types of nanosized semiconductors that are assembled by only a comparatively small amount of atoms. ${ }^{1,2}$ Their name, quantum dots (QDs), reveals a three-dimensional spatial confinement ("dot") leading to an energy discretization ("quantum"), which practically makes the control of single electrons feasible. This physical characteristic plus the large variety of QD geometries, compositions, and fabrication techniques ${ }^{3-12}$ leads to a great number of novel and promising applications in nanotechnology. ${ }^{13-16}$

Particular attention lies on the interaction of QDs with electromagnetic fields within the spectral ranges of infrared and visible light ${ }^{5,17-20}$ and comes from their potential application for optoelectronic devices, e.g. as QD infrared photodetectors. ${ }^{21,22}$ Other application in the field of energy conversion is seen for novel types of solar cells. ${ }^{23-25}$ Also the fundamental strong-field light-matter interaction between two energy levels, Rabi oscillations, ${ }^{26-35}$ is of special interest in combination with QDs for the field of quantum information processing. ${ }^{36-38}$

One recently predicted example for the interaction of electromagnetic fields with an assembly of QDs is the initiation of the inter-Coulombic decay (ICD) process. ${ }^{39,40}$ ICD was shown to be operational in a system of two QDs (and earlier in atoms ${ }^{41}$ ) as an efficient and dominant decay of a resonance state where energy is transferred between two electrons each localized in a different QD. ${ }^{39,40,42,43}$ The long-range electron interaction causes the emission of one electron from one QD into the continuum, while the other in the second QD relaxes into a lower bound state. Other decay processes such as by photon emission, electron-phonon interaction, and the Auger effect do not compete with ICD due to their much longer decay times or their energetic interdiction. ${ }^{39}$

Important for this QD-ICD, which will be the center of investigation in this paper, is an efficient preparation of the resonance state. This, we could manage via resonant excitation of the ground state by a $\pi$-pulsed infrared laser field, as we described in earlier studies. ${ }^{39,40}$ It was shown that the focus of the laser sensitively determines the impact of the second and only competing ionization channel, the direct ionization (DI). ${ }^{39}$ Focusing on the left QD only gives least contribution by DI while penetrating the full system with an unfocused 
laser causes clear disturbance of ICD through DI. Technical realization of a limited spatial focus could be by the use of shadow masks. ${ }^{38}$

The field strength dependence of the ICD initiation has already been investigated for an unfocused laser. ${ }^{40}$ The strong impact of DI including multi-photon effects made the observation of ICD possible only up to a $3 \pi$-pulse. Strong laser fields generally induce a timedependency on the electron-confinement potential, i.e. the atom's Coulomb or the QD's well potential, leading to ionization barriers alternating in time. Higher intensities cause stronger alternations making tunneling of the electrons out of their confinement increasingly likely. In addition, lowering the laser frequency $\omega$ further increases the probability for tunneling, as the time for tunneling is limited by the cycle duration $\tau=2 \pi \omega^{-1}$. Going away from this so-called quasi-static strong field regime, i.e. towards higher frequencies and lower intensities, opens up the regime of multi-photon excitations. ${ }^{44}$ There, transitions of energies corresponding to a multiple of the single-photon energy $\hbar \omega$ are possible.

The Keldysh parameter $\gamma=\omega\left(2 m_{e} V_{I E}\right)^{1 / 2}(e \eta)^{-1}$ with electron mass $m_{e}$ and charge $e$, zero-field ionization energy $V_{I E}$ and electric field strength $\eta$, serves as indicator for the preference of one of the two ionization processes. ${ }^{45}$ Tunnel ionization is more likely for values of $\gamma \ll 1\left(\gamma<0.5\right.$ required $\left.^{46}\right)$, whereas $\gamma \gg 1$ means multi-photon processes prevail. Accordingly, for our previous investigation of a $\pi$-pulse excitation in a system of GaAs $\mathrm{QDs}^{40}$ with an effective mass $m^{*}=0.063 m_{e}$, the Keldysh parameter $\gamma \approx 14$ was obtained based on $\hbar \omega=5.17 \mathrm{meV}, V_{I E}=2.31 \mathrm{meV}$ and $\eta=2.31 \times 10^{4} \mathrm{Vm}^{-1}$, which indicates the multi-photon regime. Hence, we expect tunneling to become dominant only for pulses of field strengths about 28 times larger than for the $\pi$-pulse. To reach that regime the intensity needed to be three orders of magnitude higher than for the $\pi$-pulse, which is, however, clearly out of the range of observation.

To investigate more deeply on the effect of multi-photon processes during the resonant excitation, we theoretically study the electron dynamics for the complete excitation and decay process in the QD pair. We stick to a focused laser to obtain a larger field strength window for our observations by systematically quenching DI. A space-resolved wavefunction ansatz is applied by means of the antisymmetrized multiconfiguration time-dependent Hartree method (MCTDH). ${ }^{47,48}$ When performing grid-based dynamics as implemented in the Heidelberg MCTDH program, ${ }^{49,50}$ special care has to be taken regarding the avoidance of back-scattering of unbound electrons at the grid ends. Hence, oftentimes complex absorbing 
potentials $(\mathrm{CAP})^{51-53}$ are applied outside of the bound region to absorb electron density of delocal continuum states. It is obvious that CAPs may not only help, but also disturb the system's wavefunction dynamics. Even when only a single particle is absorbed, it will lead to a decrease of the wavefunction norm to zero, such that remaining particles are not defined anymore. ${ }^{54,55}$ Further, if the CAP reaches too far into the region of localized states it can artificially enhance the continuum state population by pulling bound electrons. In addition, the CAP strength must be chosen optimally, otherwise particle might pass the CAP or even be reflected by it.

What seemed a technicality at first, turned out to sensitively influence the analysis and interpretation of our dynamics results. We already found that ICD rates are independent whether calculating with or without $\mathrm{CAP},{ }^{40}$ but we also saw irregularities in the dynamics caused by the CAP. Firstly, compared to calculations on a grid without CAP the resonance state population is too low. ${ }^{40}$ Secondly, the single-electron state populations of the twolevel (left) QD are displayed incorrectly due to missing continuum terms. ${ }^{39,40}$ However, when scanning fields strengths, we were dependent on using CAPs while sacrificing the understanding of the system based on accurate populations. To overcome this limitation, we developed a more accurate analysis for CAP-free calculations performed on a longer grid during rather short, but sufficient propagation times. With the abscence of the CAP it is now also possible to determine the populations of multiple continuum configurations, which facilitates our understanding especially of DI and multi-photon processes in dependence on the laser focus and intensity.

This paper is organized as follows: In Sec. II we introduce the QD pair model. The electron dynamics is described in Sec. III for two complementary wavefunction approaches. The results can be found in Sec. IV. Sec. IV A shows the $\pi$-pulse induced QD-ICD comparing laser foci and usage of a CAP. The field strength scan and $n \pi$-pulse excitations are presented for a focused laser and CAP-free calculations in Sec. IV B. The conclusion can be found in Sec. V.

\section{MODEL}

For the model potential of two singly negatively charged and uncoupled QDs we use the double-well system that has been investigated in previous studies. ${ }^{39,40,43,56,57}$ The QDs are 
chosen to be made of GaAs with the material specific effective electron mass $m^{*}=0.063 m_{e}$ and dielectric constant $\kappa=12.9,{ }^{58}$ that enter into the calculations.

In the $x$ - and $y$-direction the electronic motion shall be strongly confined by harmonic oscillator potentials. In the resulting quasi one-dimensional (q1d) system the QD pair is modeled by two negative Gaussian potentials along the pairing z-axis,

$$
\hat{V}_{\mathrm{QD}}(z)=-\sum_{j=L, R} D_{j} e^{-4 \ln (2)\left(\left(\hat{z}-z_{j}\right) / r_{j}\right)^{2}},
$$

with the sum running over the two QDs, which we call left $(\mathrm{L})$ and right $(\mathrm{R})$. The QD centers at $z_{L}=-\tilde{R} / 2$ and $z_{R}=\tilde{R} / 2$ are defined via the inter-QD distance $\tilde{R}=86.68 \mathrm{~nm}$. Further parameters defining the system are the depths $D_{L}=10.30 \mathrm{meV}, D_{R}=8.24 \mathrm{meV}$ and the full widths at half maximum $r_{L}=36.08 \mathrm{~nm}$ and $r_{R}=18.04 \mathrm{~nm}$.

The Hamiltonian of the time-independent q1d two-electron system,

$$
\hat{H}_{\mathrm{el}}=\hat{h}_{1}+\hat{h}_{2}+\hat{V}_{\mathrm{Coul}}^{\mathrm{q} 1 \mathrm{~d}}\left(z_{12}\right)
$$

contains the sum over the single-electron Hamiltonians,

$$
\hat{h}_{i}=-\frac{1}{2} \frac{\partial^{2}}{\partial z_{i}^{2}}+\hat{V}_{\mathrm{QD}}\left(z_{i}\right),
$$

for $i=1,2$ with corresponding discrete $|\lambda\rangle$ and continuum eigenstates $|\varepsilon\rangle$. For the above chosen parameters we find in particular two energy levels for the left QD $\left(\lambda=L_{0}, L_{1}\right)$ and one for the right $\left(\lambda=R_{0}\right)$ with the energetic order $E_{L_{0}}^{1 e}<E_{R_{0}}^{1 e}<E_{L_{1}}^{1 e}$. Above these energies the spectrum is continuous with energies $\varepsilon \in[0, \infty)$. In Eq. (2) the Coulomb interaction between both electrons, which is for three dimensions well known to be defined as $\left|\mathbf{r}_{1}-\mathbf{r}_{2}\right|^{-1}$ with $\mathbf{r}_{i=1,2}$ being the electrons' spatial coordinates, has been converted into a q1d form,

$$
\hat{V}_{\mathrm{Coul}}^{\mathrm{q} 1 \mathrm{~d}}\left(z_{12}\right)=\sqrt{\frac{\pi}{2}} e^{\hat{z}_{12}^{2} / 2} \operatorname{erfc}\left(\hat{z}_{12} / \sqrt{2}\right)
$$

with the inter-electron distance $\hat{z}_{12}=\left|\hat{z}_{1}-\hat{z}_{2}\right|{ }^{56,59,60}$ The solution to the respective timeindependent Schrödinger equation for the two-electron system,

$$
\hat{H}_{\mathrm{el}}|\alpha\rangle=E_{\alpha}^{2 e}|\alpha\rangle
$$

yields the eigenstates $|\alpha\rangle$ with the corresponding eigenenergies $E_{\alpha}^{2 e}$. The index $\alpha=$ $\left\{\lambda \lambda^{\prime}, \lambda \varepsilon, \varepsilon \varepsilon^{\prime}\right\}$ denotes the possible configurations with the three different cases of either 
both electrons occupying bound QD levels $\left(\lambda \lambda^{\prime}\right)$, both occupying continuum levels $\left(\varepsilon \varepsilon^{\prime}\right)$, or a mixture of both $(\lambda \varepsilon)$.

Here, we deliberately chose the more intensely investigated triplet electron configuration, i.e. same-spin electrons that require antisymmetrized spatial eigenstates. ${ }^{39,40}$ This results in a smaller number of bound states compared to spin-singlet states, namely $\left|L_{0} R_{0}\right\rangle,\left|L_{1} R_{0}\right\rangle$ and $\left|L_{0} L_{1}\right\rangle$, where $\left|L_{0} R_{0}\right\rangle$ is the energetic ground state. For observing ICD, solely the resonance state $\left|L_{1} R_{0}\right\rangle$ is relevant. As the electrons are far apart, exchange is small and the decay behavior of spin-singlet and spin-triplet states has been shown to give very similar decay rates,${ }^{60}$ so that one could even work without antisymmetrization after the preparation of our appropriate initial state.

The population of $\left|L_{0} L_{1}\right\rangle$ with both electrons in the left QD is negligibly small during the whole process, since on the one hand we start from the ground state $\left|L_{0} R_{0}\right\rangle$ and on the other hand tunneling is ruled out as the localized single-electron states do not overlap and excitation intensities for the $n \pi$-pulses will be low enough. Therefore, the terms related to this state are omitted in all our following considerations.

\section{ELECTRON DYNAMICS}

The process under investigation is depicted in Fig. 1. Initially, the system is in its twoparticle ground state $\left|L_{0} R_{0}\right\rangle$ with one electron per QD. The resonant excitation by a $\pi$-pulse of energy $\omega_{\mathrm{RES}}=E_{L_{1} R_{0}}^{2 e}-E_{L_{0} R_{0}}^{2 e}$ causes the complete depopulation of the ground state and inversion into $\left|L_{1} R_{0}\right\rangle$ which corresponds to $1 / 2$ of a Rabi cycle. ${ }^{26}$ A single decay channel exists for the resonance state which is ICD into a continuum state $\left|L_{0} \varepsilon\right\rangle$ of equal energy. The $L_{0}$-continuum state can further be populated through DI of the ground state's $R_{0}$ electron. Besides these two single-photon processes that yield states of energy $E_{L_{0} R_{0}}^{2 e}+\omega$, numerous

processes which result in continuum states of energies $E_{L_{0} R_{0}}^{2 e}+m \omega$ with $m \in \mathbb{N}, m>1$, are possible and shall be studied here as well. Those up to $m=2$ are explicitly depicted in Fig. 1 . The multi-photon excited states with $m>1$ are either prepared via direct transitions with a multiple of the excitation energy or via step-wise excitations by consecutive single-photon processes as we will elucidate. 


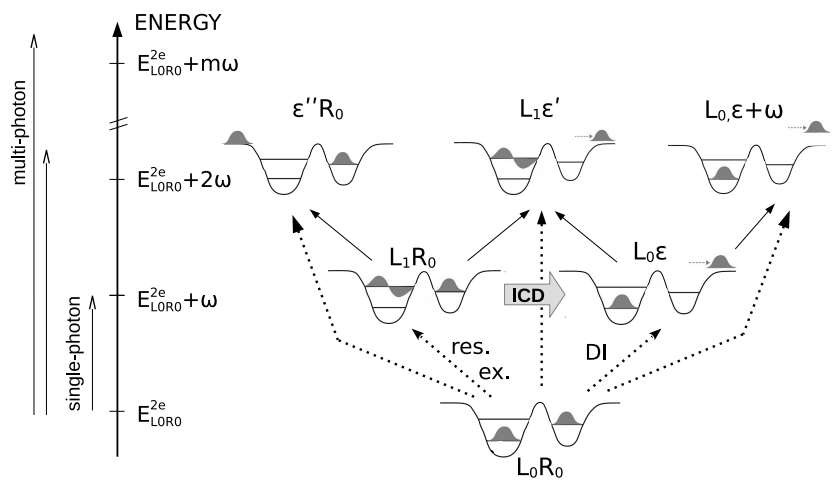

FIG. 1. The light-induced ICD process in the two-electron QD pair with the ground state $\left|L_{0} R_{0}\right\rangle$ as initial state here shown for a laser directed on both QDs. After resonant excitation (res. ex.) by a short laser pulse the resonance state $\left|L_{1} R_{0}\right\rangle$ decays via ICD into a continuum state $\left|L_{0} \varepsilon\right\rangle$ of same energy. Besides ICD, the direct ionization (DI) of the ground state by the laser is possible. During the excitation, states of higher energies $E_{L_{0} R_{0}}^{2 e}+m \omega$ with $m>1$ can be populated via multi-photon processes, either via direct $m$-photon excitation (dotted arrows), or $m$ consecutive single-photon processes (short arrows).

The time-dependent wavefunction as solution to the Schrödinger equation,

$$
i \frac{\partial}{\partial t}|\Psi(t)\rangle=\hat{H}(t)|\Psi(t)\rangle
$$

yields the necessary information on the electron dynamics. The Hamiltonian

$$
\hat{H}(t)=\hat{H}_{\mathrm{el}}+\hat{H}_{\text {field }}(t)
$$

is a combination of the time-independent electronic part $\hat{H}_{\mathrm{el}}$ (Eq. (2)) and a time-dependent term for the electron-field interaction, which is represented in the semiclassical dipole approximation,

$$
\hat{H}_{\text {field }}(t)=-\mathcal{E}_{z}(t) \hat{\mu}_{z}
$$

The electric dipole operator $\hat{\mu}_{z}=-\sum_{i} \hat{z}_{i}$ sums over the two electrons. The time-dependent electric field $\mathcal{E}_{z}(t)$ of the $z$-polarized resonant laser pulse,

$$
\mathcal{E}_{z}(t)=\eta \cos \left(\omega_{\mathrm{RES}} t\right) \sin ^{2}\left(\frac{\pi}{t_{\text {pulse }}} t\right) \Theta\left(t_{\text {pulse }}-t\right),
$$

is defined by the field strength $\eta$, the pulse duration $t_{\text {pulse }}$ and the photon energy $\omega_{\mathrm{RES}}$. The intensity of the laser field relates to the field strength via $I=\eta^{2}(8 \pi \alpha)^{-1}$ with the fine 


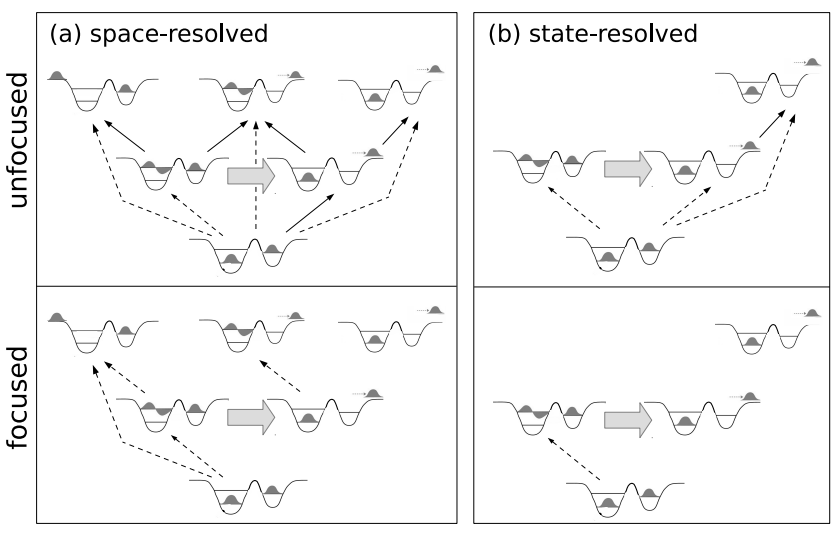

FIG. 2. The relevance of states is shown depending on the method. The main method ((a) space-resolved) represents all states with equal accuracy, whereas the complementary method ((b) state-resolved) contains only the two localized states, $\left|L_{0} R_{0}\right\rangle$ and $\left|L_{1} R_{0}\right\rangle$, and the $L_{0}$-continuum states. For both methods the excitation pathways are depicted by the arrows for the two laser foci. Upper panels show the case of the laser pulse directed on the full system while the lower ones are for a focused laser on the left QD only.

structure constant $\alpha$ as inverse of the speed of light in vacuum. To adjust the focus of the laser, we utilize a Heaviside step function and modify Eq. (8) accordingly,

$$
\hat{H}_{\text {field }}^{\prime}(t)=\Theta\left(z-z_{A}\right) \hat{H}_{\text {field }}(t) \Theta\left(z_{B}-z\right) .
$$

Thus, by choosing $z_{A}$ and $z_{B}$ as left and right end points of the grid the laser is applied on the full system and all excitation pathways are possible (cf. upper of Fig 2(a)). To focus solely on the left QD the right cutoff point must be changed to the center between the QDs, i.e. $z_{B}=0 \mathrm{~nm}$. In this case, we expect the DI of the right QD to be minimized which will reduce the number of populated states (cf. lower of Fig 2(a)).

For continuous resonant excitations in a two-level system the populations of the ground state (GS) and the resonance state (RS) are described in terms of Rabi oscillations and are given by the following time-dependent expressions, ${ }^{61}$

$$
\begin{aligned}
& P_{\mathrm{GS}}(t)=\cos ^{2}\left(\frac{\Omega_{\mathfrak{R}} t}{2}\right), \\
& P_{\mathrm{RS}}(t)=\sin ^{2}\left(\frac{\Omega_{\mathfrak{R}} t}{2}\right) .
\end{aligned}
$$

The Rabi frequency $\Omega_{\mathfrak{R}}$ is defined by the cycle duration $T=2 \pi \Omega_{\mathfrak{R}}^{-1}$. For our two-electron QD pair system, Rabi oscillations compete with the decay of the resonance state via ICD 
into a continuum state as well as with direct ionization processes. The ground and resonance state populations can therefore be described by the modified equations,

$$
\begin{aligned}
& P_{\mathrm{L} 0 \mathrm{R} 0}(t)=e^{-\left(\Gamma_{\mathrm{ICD}}+\Gamma_{\mathrm{ion}, \eta}\right) t} \cos ^{2}\left(\frac{\Omega_{\mathfrak{R}}(t) t}{2}\right), \\
& P_{\mathrm{L} 1 \mathrm{R} 0}(t)=e^{-\left(\Gamma_{\mathrm{ICD}}+\Gamma_{\mathrm{ion}, \eta}\right) t} \sin ^{2}\left(\frac{\Omega_{\mathfrak{R}}(t) t}{2}\right) .
\end{aligned}
$$

The exponential factor now introduces the decay via ICD with rate $\Gamma_{\text {ICD }}$ as well as DI with the field-dependent rate $\Gamma_{\text {ion, }, \eta}=(\eta / 2)^{2} \times \Gamma_{\text {ion. }}$. Furthermore, the Rabi frequency is time-dependent and given by the following expression,

$$
\Omega_{\mathfrak{R}}(t)=\mu \eta g(t),
$$

with the transition dipole moment $\mu=2\left\langle L_{1} R_{0}|z| L_{0} R_{0}\right\rangle$. The time-dependence of the Rabi frequency is resulting from the envelope function $g(t)=\sin ^{2}\left(\pi t / t_{\text {pulse }}\right)$ used for the shape of the pulse in Eq. (9). If we demand all population within the QDs to be excited from the ground state into the resonance state by the end of the pulse, it would require the fulfillment of,

$$
\int_{0}^{t_{\mathrm{pulse}}} \Omega_{\mathfrak{R}}(t) d t=\pi,
$$

according to Eqs. (14) and (15). Thus, we obtain a relation for the field strength in dependence on the pulse length describing the so-called $\pi$-pulse condition, namely

$$
\eta_{\pi}=\frac{2 \pi}{\mu t_{\text {pulse }}}
$$

Choosing short pulse lengths will result in a high field strength in favor for DI. For small field strengths a long pulse length is needed, which in contrary gives enough time for the resonance state to decay via ICD. The optimal laser parameters are found for when the resonance state is populated to a maximum. However, the ionization processes cannot be completely avoided, such that the maximum of the resonance state will never reach a value of one. It is further constraint by the transition dipole moment $\mu$.

\section{A. Space-resolved representation}

For a representation of the wavefunction in spatial coordinates we make use of the multiconfiguration time-dependent Hartree (MCTDH) method ${ }^{47,48}$ as implemented in the Heidelberg MCTDH package. ${ }^{49,50}$ The wavefunction for a system with two degrees of freedom $z_{i}$ 
$(i=1,2)$ is described by a sum of Hartree products,

$$
\Psi^{\mathrm{MCTDH}}\left(z_{1}, z_{2}, t\right)=\sum_{j_{1}}^{N_{1}} \sum_{j_{2}}^{N_{2}} A_{j_{1} j_{2}}(t) \psi_{j_{1}}^{(1)}\left(z_{1}, t\right) \psi_{j_{2}}^{(2)}\left(z_{2}, t\right)
$$

with time-dependent, and for the spin-triplet case, antisymmetric coefficients $A_{j_{1} j_{2}}(t)=$ $-A_{j_{2} j_{1}}(t)$, and likewise time-dependent single-particle functions (SPFs) $\psi_{j_{i}}^{(i)}\left(\mathbf{r}_{i}, t\right)$, with index $j_{i}$ and number of SPFs $N_{i}$ for the $i$-th degree of freedom.

Both, the SPFs and the coefficients are propagated according to the MCTDH equations of motion, which are derived from the Dirac-Frenkel time-dependent variational principle, i.e. $\left\langle\delta \Psi\left|\hat{H}-i \partial_{t}\right| \Psi\right\rangle=0$. In a preceding block improved relaxation for the time-independent system, $\hat{H}=\hat{H}_{\mathrm{el}}$, the eigenstates are obtained, i.e the ground and resonance state amongst many continuum states. The ground state is then propagated in real time, where the initial wave packet interacts with the external time-dependent field and undergoes simultaneously ICD and possibly direct photoionization.

For result analysis, the populations of the localized two-electron states $|\alpha\rangle$ with $\alpha=$ $L_{0} R_{0}, L_{1} R_{0}$ are obtained from the expectation value of the projection operator $\hat{P}_{\alpha}=|\alpha\rangle\langle\alpha|$,

$$
\begin{aligned}
P_{\alpha}(t) & =\left\langle\Psi(t)\left|\hat{P}_{\alpha}\right| \Psi(t)\right\rangle \\
& =|\langle\alpha \mid \Psi(t)\rangle|^{2} .
\end{aligned}
$$

The one-electron bound state populations are calculated equally as,

$$
P_{\lambda}(t)=2\left|\left\langle\lambda\left|\mathbb{1}_{2}\right| \Psi(t)\right\rangle\right|^{2}
$$

with $\lambda=L_{0}, L_{1}, R_{0}$. The prefactor two accounts for the two identical electrons. For inclusion of the second coordinate the projection on the two-particle identity is necessary,

$$
\begin{aligned}
\mathbb{1}_{2}= & \frac{1}{2} \sum_{\lambda \lambda^{\prime}}\left|\lambda \lambda^{\prime}\right\rangle\left\langle\lambda \lambda^{\prime}\left|+\sum_{\lambda} \int d \varepsilon\right| \lambda \varepsilon\right\rangle\langle\lambda \varepsilon| \\
& +\frac{1}{2} \iint d \varepsilon d \varepsilon^{\prime}\left|\varepsilon \varepsilon^{\prime}\right\rangle\left\langle\varepsilon \varepsilon^{\prime}\right| .
\end{aligned}
$$

To evaluate the expressions of the overlap of one- and two-electron states, one may rewrite the latter by products of single-electron states,

$$
\left|\lambda \lambda^{\prime}\right\rangle=\frac{1}{\sqrt{2}}\left(\left|\lambda^{\prime}\right\rangle|\lambda\rangle-|\lambda\rangle\left|\lambda^{\prime}\right\rangle\right)
$$


which holds also for the continuum states. Thus, by inserting Eq. (21) with the usage of Eq. (22), Eq. (20) turns into

$$
P_{\lambda}(t)=\sum_{\lambda^{\prime}} P_{\lambda^{\prime} \lambda}(t)+\int d \varepsilon P_{\lambda \varepsilon}(t),
$$

where $P_{\lambda^{\prime} \lambda}(t)=P_{\lambda \lambda^{\prime}}(t)$ or $P_{\lambda \varepsilon}(t)=P_{\varepsilon \lambda}(t)$ is valid. Eq. (23) yields the following relations for the single-electron state populations expressed via the two-electron states,

$$
\begin{aligned}
& P_{L_{0}}(t)=P_{L_{0} R_{0}}(t)+P_{L_{0} C}(t), \\
& P_{L_{1}}(t)=P_{L_{1} R_{0}}(t)+P_{L_{1} C}(t), \\
& P_{R_{0}}(t)=P_{L_{0} R_{0}}(t)+P_{L_{1} R_{0}}(t)+P_{C R_{0}}(t) .
\end{aligned}
$$

Here, we introduced the short notation for the continuum terms $P_{\lambda C}(t)=\int d \varepsilon P_{\lambda \varepsilon}(t)$ or for two continuum electrons $P_{C C}(t)=\iint d \varepsilon d \varepsilon^{\prime} P_{\varepsilon \varepsilon^{\prime}}(t)$. The relations Eqs. (24)-(26) provide the possibility to determine the different continuum state populations.

The effective Coulomb potential Eq. (4) has been converted into MCTDH form in an exact expansion, i.e. a sum of products of single particle potentials, by employment of the POTFIT program. ${ }^{49,62,63}$ For the SPFs the Runge-Kutta integrator of order eight was applied, for the A-coefficients a short-time Lanczos integrator. The effectively realized propagation time step of the wavefunction was about 0.064 ps.

We compare calculations on a short and a long grid with 140 and 980 sine DVR ${ }^{49,64}$ points within an interval of $[-542 \mathrm{~nm}, 542 \mathrm{~nm}]$ and [-3792 nm, $3792 \mathrm{~nm}]$, respectively. The constant mesh density of $\rho_{z}=0.129 \mathrm{pts} \mathrm{nm}^{-1}$ has been approved in our previous studies being sufficient to treat the highest excited continuum states encountered here. ${ }^{57}$ For the calculations on the short grid a CAP of 4 th-order and strength $\eta_{\mathrm{CAP}}=8.6997 \times 10^{-6}$ has been applied left and right from the QD pair at $z= \pm 325 \mathrm{~nm}$. The long grid is CAP-free.

When treating Hermitian systems, the norm of the wavefunction $|\Psi(t)|^{2}$ equals unity at all times. In the case of our CAP-free system, this means

$$
\left\langle\Psi(t)\left|\mathbb{1}_{2}\right| \Psi(t)\right\rangle=P_{L_{0} R_{0}}(t)+P_{L_{1} R_{0}}(t)+P_{\text {cont }}(t)=1 .
$$

As shown here, when inserting the identity of Eq. (21) the norm can be expressed as sum over all two-electron eigenstate populations consisting of the two available localized states $\left|L_{0} R_{0}\right\rangle,\left|L_{1} R_{0}\right\rangle$, and the sum of continuum state populations,

$$
P_{\text {cont }}(t)=P_{L_{0} C}(t)+P_{L_{1} C}(t)+P_{C R_{0}}(t)+P_{C C}(t) .
$$


Applying a CAP makes the system non-Hermitian. The absorption of continuum electrons causes a decrease of the norm during propagation, i.e. $|\Psi(t)|^{2}<1$ for times $t>t_{\mathrm{CAP}}$, where $t_{\mathrm{CAP}}=t_{\text {ion }}+t_{\text {fly }}$ is the time the electron needs to undergo ionization $\left(t_{\text {ion }}\right)$ plus the time of flight until it reaches the CAP $\left(t_{\mathrm{fly}}\right)$. Hence, the norm is reduced by the amount of continuum states $P_{\text {cont }}(t)$ with a time shift $t_{\text {fly }}$ and the following relation must be valid,

$$
\begin{aligned}
\left|\Psi\left(t+t_{\text {fly }}\right)\right|^{2} & =1-P_{\text {cont }}(t) \\
& =P_{L_{0} R_{0}}(t)+P_{L_{1} R_{0}}(t),
\end{aligned}
$$

where in the second line we made use of Eq. (27). According to Eq. (29), for times after the pulse the norm decreases together with the resonance state population due to ICD. As a consequence, for a $\pi$-pulse, which leaves no population in the ground state, the norm decreases towards zero.

\section{B. State-resolved representation}

Besides the space-resolved method (Sec. III A), a second approach has been introduced ${ }^{40}$ allowing for much smaller computation times and which we want to apply here again. In this, the wavefunction is resolved into its individual eigenstates, ${ }^{65,66}$ but the number of states that are actually available is constrained to the ones relevant for the ICD process, i.e. the ground and resonance state $\left|L_{0} R_{0}\right\rangle$ and $\left|L_{1} R_{0}\right\rangle$, as well as the range of continuum states $\int d \varepsilon\left|L_{0} \varepsilon\right\rangle\left\langle L_{0} \varepsilon\right|$. The latter include the states resulting from the ICD decay and they are naturally also the states subject to DI, cf. upper panel of Fig. 2(b).

The wavefunction is fully described by expansion in the orthonormal basis of the twoelectron eigenstates. For this purpose $|\Psi(t)\rangle$ is multiplied by the identity Eq. (21). Under the constraint of the available states, renamed as initial state $|i\rangle=\left|L_{0} R_{0}\right\rangle$, decaying state $|d\rangle=$ $\left|L_{1} R_{0}\right\rangle$ and final state $\left|f_{\varepsilon}\right\rangle=\left|L_{0} \varepsilon\right\rangle$, we obtain the simplified state-resolved wavefunction,

$$
\left|\Psi^{\text {state }}(t)\right\rangle=a_{i}(t)|i\rangle+a_{d}(t)|d\rangle+\int d \varepsilon a_{f_{\varepsilon}}(t)\left|f_{\varepsilon}\right\rangle
$$

where the time-dependent coefficients $a_{\alpha}(t)=\langle\alpha \mid \Psi(t)\rangle$ with $\alpha=i, d, f_{\varepsilon}$, have been intro-

duced. The populations of the two-electron states are directly accessible via $P_{\alpha}(t)=\left|a_{\alpha}(t)\right|^{2}$. The Schrödinger equation (6) with the state-resolved wavefunction Eq. (30) and the Hamil- 
tonian Eq. (7) yields the following equations of motion,

$$
\begin{aligned}
i \dot{a}_{i}(t)= & \left(E_{i}-\frac{i}{2} \Gamma_{\mathrm{ion}}\left|\mathcal{E}_{z}(t)\right|^{2}\right) a_{i}(t) \\
& +\left(\mu_{\mathrm{ex}}^{*}-\frac{i}{2} \gamma^{*}\right) \mathcal{E}_{z}(t) a_{d}(t) \\
i \dot{a}_{d}(t)= & \left(\mu_{\mathrm{ex}}-\frac{i}{2} \gamma\right) \mathcal{E}_{z}(t) a_{i}(t)+\left(E_{d}-\frac{i}{2} \Gamma_{\mathrm{ICD}}\right) a_{d}(t), \\
i \dot{a}_{f_{\varepsilon}}(t)= & \mu_{\mathrm{ion}} \mathcal{E}_{z}(t) a_{i}(t)+V a_{d}(t)+E_{f_{\varepsilon}} a_{f_{\varepsilon}}(t),
\end{aligned}
$$

as has been derived in detail previously. ${ }^{40}$ The products of electric field $\mathcal{E}_{z}(t)$ and transition dipole moments describe the light-matter interaction. The two relevant transitions caused by the field are via the resonant photoexcitation with the corresponding dipole moment $\mu_{\mathrm{ex}}=2\langle d|z| i\rangle$ and the direct photoionization with $\mu_{\mathrm{ion}}=2\left\langle f_{\varepsilon}|z| i\right\rangle$, from which the fieldindependent ionization rate can be calculated as $\Gamma_{\text {ion }}=2 \pi\left|\mu_{\text {ion }}\right|^{2}$. The decay via ICD from $|d\rangle$ to $\left|f_{\varepsilon}\right\rangle$ induced by Coulomb interaction is contained in the transition matrix element $V=\left\langle f_{\varepsilon}\left|V_{\text {Coul }}^{\mathrm{q} 1 \mathrm{~d}}\right| d\right\rangle$, which defines the ICD rate as $\Gamma_{\mathrm{ICD}}=2 \pi|V|^{2}$. The rate $\gamma=2 \pi V^{*} \mu_{\text {ion }}$ describes the mixture of the two ionization pathways, i.e. ICD and DI. Note that the radiative decay from $|d\rangle$ to $|i\rangle$ is negligible, since it was found to be three orders of magnitude slower than all other relevant transitions. ${ }^{43}$

Eqs. (31)-(33) were solved numerically with a non-commercial Fortran program. ${ }^{40}$ Most of the required parameters are determined in advance from a MCTDH relaxation and propagation calculation for the CAP-free system, namely the eigenenergies $E_{i}=-9.46 \mathrm{meV}$ and $E_{d}=E_{f_{\varepsilon}}=-4.28 \mathrm{meV}, \Gamma_{\mathrm{ICD}}=4.09 \times 10^{-3} \mathrm{meV}\left(6.22 \times 10^{-3} \mathrm{ps}^{-1}\right)$ and $\mu_{\mathrm{ex}}=1.67 \times$ $10^{-27} \mathrm{Cm}$, and further the field parameters for the $\pi$-pulse, $\omega_{\mathrm{RES}}=5.18 \mathrm{meV}, t_{\text {pulse }}=14.1 \mathrm{ps}$ and $\eta_{\pi}=2.82 \times 10^{4} \mathrm{Vm}^{-1}$, corresponding to an intensity of $I=1.37 \times 10^{3} \mathrm{Wcm}^{-2}$. However, the ionization rate $\Gamma_{\text {ion }}$ cannot be determined straightforwardly, since the exact final state is impossible to find due to the finite resolution of the continuum from the MCTDH relaxation calculations. Still, $\Gamma_{\text {ion }}$ can be obtained from a fit of the state- to the space-resolved results (Sec. IV B). The focus of the laser is here determined by the value of $\Gamma_{\text {ion. }}$. For an unfocused laser the rate is higher since more DI of the right QD takes place in comparison to the focused laser.

In comparison to the space-resolved method which treats the continuum at equally high accuracy as the bound states, the state-resolved method yields a description of the continuum of limited accuracy. However, comparing the results of the two wavefunction approaches will 
serve us as a method to predict the importance of multi-photon processes.

\section{RESULTS}

\section{A. $\pi$-pulse induced ICD}

At first, we are going to study the impact of the laser focus on the ICD process in the two-electron QD pair. This is done by evaluating the electron dynamics with the help of the time-dependent populations of the localized and continuum states under a $\pi$-pulse excitation and thereafter. The MCTDH method is applied and we put particular emphasis on the evaluation of the influence of the CAP on the calculations.

The $\pi$-pulse laser parameters were determined according to Eq. (17) with the calculated transition dipole moment $\mu=1.67 \times 10^{-27} \mathrm{Cm}$ under a yield maximization of the resonance state population. For the CAP-free calculations, the highest population has been found for the combination of pulse length $t_{\text {pulse }}=14.1 \mathrm{ps}$ and field strength $\eta_{\pi}=2.82 \times 10^{4} \mathrm{Vm}^{-1}$. To enable a better comparison, the same laser parameters were applied for the calculations with CAP.

The resonant excitation energies have been determined from the preceding relaxation calculations for both grids as $\omega_{\mathrm{RES}}^{\mathrm{CAP}}=5.175 \mathrm{meV}$ (short grid with CAP) and $\omega_{\mathrm{RES}}=5.179 \mathrm{meV}$ (long grid without CAP), thus being in the range of infrared light. They differ slightly, because the relaxed state energies are affected by the grid size.

The results are presented in Figs. 3 and 4. Panels 3(a)-(d) show the time-dependence of the one- and two-electron bound and localized state populations, $P_{L_{0}}, P_{L_{1}}$ and $P_{R_{0}}$, as well as $P_{L_{1} R_{0}}$ and $P_{L_{0} R_{0}}$, as a result of the projections of Eqs. (19) and (20). Panels (a)+(b) belong to calculations performed on the short grid with CAP, $(c)+(d)$ on the long grid without CAP. In (a)+(c) the results for the focused laser are displayed, $(b)+(d)$ for the full laser penetration of both QDs. We specifically compare the resonance state populations for calculations on the two different grids either with or without CAP for the focused (Fig. 3(e)) or unfocused (f) pulse. This gives information on the impact of the CAP on the dynamics.

In Fig. 4 the continuum state populations $P_{L_{0} C}, P_{L_{1} C}$ and $P_{C R_{0}}$ are presented as functions of time for both laser foci and for calculations on the long grid without CAP. These are obtained using Eqs. (24)-(26) together with the results of the bound and localized state 

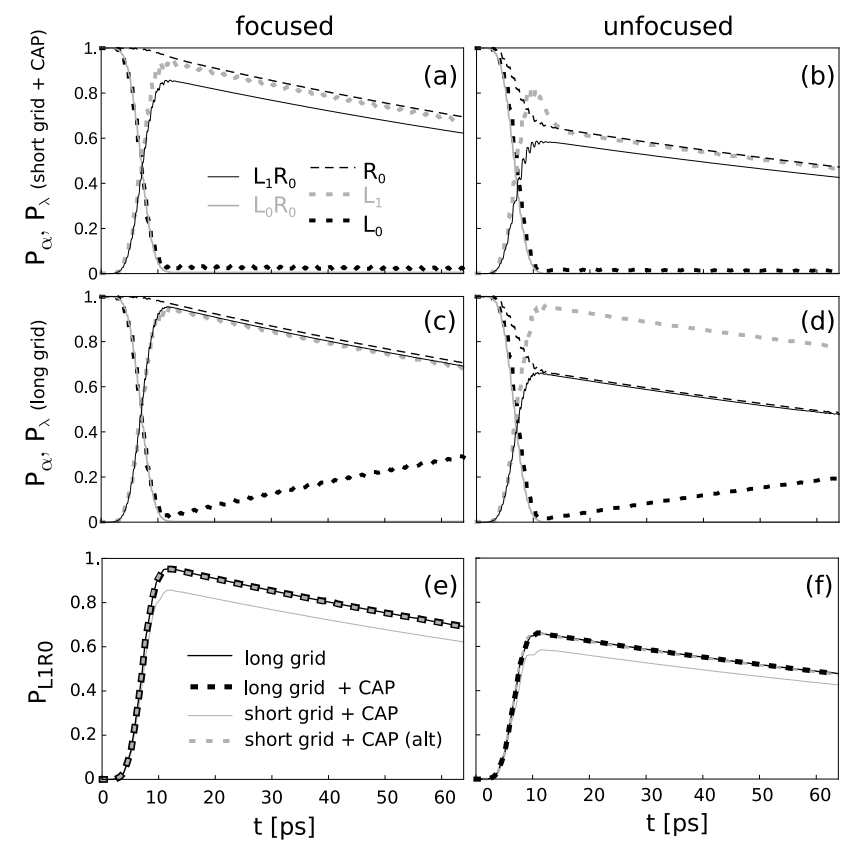

FIG. 3. The $\pi$-pulse induced ICD process in the QD pair with pulse length $t_{\text {pulse }}=14.1$ ps. Panels (a)-(d) show populations of the single-electron levels $P_{\lambda}$ (dashed lines) and the two-electron localized states $P_{\alpha}$ (solid lines) as functions of time. Calculations were performed by usage of the space-resolved method on the short grid with CAP $(\mathrm{a})+(\mathrm{b})$ and on the long grid without CAP $(\mathrm{c})+(\mathrm{d})$. Panels (e)+(f) display the resonance state populations obtained from the projections for different grid-CAP combinations, as well as an alternative (alt) calculation for the short grid with CAP. Panels $(a)+(c)+(e)$ show results for a laser penetration of the left QD only, $(b)+(d)+(f)$ are for the laser acting on the full system.

projections of Eqs. (19) and (20). Finally, the population $P_{\mathrm{CC}}(t)$, i.e. the sum over those configurations where both electrons are in the continuum, can be deduced by making use of the constancy of the norm Eq. (27) together with Eq. (28).

First of all, we will fully describe the dynamics of the excitation and decay process exemplarily for the CAP-free system for calculations on the long grid and a laser focused on the left QD only, cf. Figs. 3(c)+4(a). In our understanding, this combination provides most comprehension on the process for the three reasons that continuum state populations can be determined, propagation times are sufficiently long before backscattering disturbs the dynamics within the QDs, and direct ionization of the right QD is assumably minimized. Initially, at $t=0 \mathrm{ps}$, the system is in its ground state, thus $\left|L_{0} R_{0}\right\rangle$ as well as the 
(a) focused

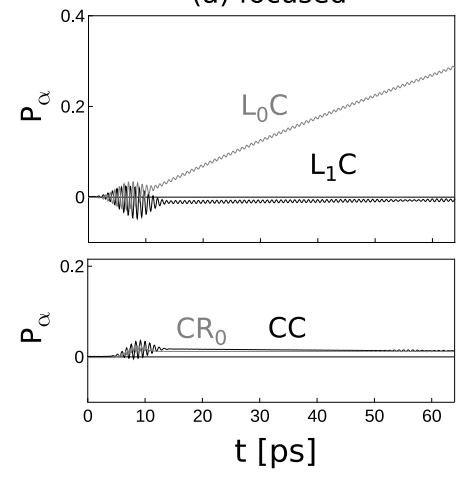

(b) unfocused

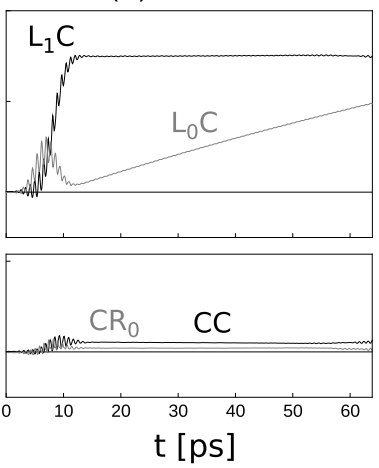

FIG. 4. Population $P_{\alpha}$ of the two-electron continuum states $|\alpha\rangle=\left|L_{0} C\right\rangle,\left|L_{1} C\right\rangle,\left|C R_{0}\right\rangle$ and $|C C\rangle$ as a function of time for the $\pi$-pulse calculations without CAP and (a) a focused and (b) an unfocused laser.

single-electron states $\left|L_{0}\right\rangle$ and $\left|R_{0}\right\rangle$ are fully populated while all other states contain zero population. During the laser pulse a population inversion from the ground to the resonance state $\left|L_{1} R_{0}\right\rangle$ can be observed with an equal inversion from $\left|L_{0}\right\rangle$ to $\left|L_{1}\right\rangle$. The inversion is not immediately visible from the start of the pulse at $t=0 \mathrm{ps}$, but with a delay of about 3 ps when the pulse is strong enough. The ground state population reaches zero and the resonance state population its maximum of 0.95 at $11.5 \mathrm{ps}$, thus 2.6 ps prior to the end of the pulse. The latter can be ascribed to the fact that ICD is initiating the decay of $\left|L_{1} R_{0}\right\rangle$ into $\left|L_{0} C\right\rangle$ already during the laser excitation. This can be seen from the decrease of the $\left|R_{0}\right\rangle$-curve starting at 6 ps. As a consequence, by the end of the pulse already $4 \%$ of the population can be found in $\left|L_{0} C\right\rangle$. Hence, the resonance state is never fully populated and the maximum is reached before the end of the pulse. On the other hand, during the excitation process, $P_{\mathrm{L}_{0} \mathrm{C}}$ remains generally small, which means a low probability for DI of the right QD. After the pulse, the ground state remains unpopulated, while finally the decay of the resonance state solely via ICD can be observed. The ICD rate can be obtained from the exponential decay according to $P_{L_{1} R_{0}}(t) \propto e^{-\Gamma_{\mathrm{ICD}} t}$. Vice versa, an increase of $\left|L_{0} C\right\rangle$ proportional to $1-e^{-\Gamma_{\mathrm{ICD}} t}$ can be seen. Hence, the $\left|L_{0}\right\rangle$ population starts to increase again, while $\left|L_{1}\right\rangle$ and $\left|R_{0}\right\rangle$ decay with the resonance state. Contributions from direct ionizations of the left or both QDs are visible, but marginal (cf. $\left|C R_{0}\right\rangle$ - and $|C C\rangle$-population in the lower panel of Fig. 4(a)). Strong oscillations of the continuum state populations reaching into negative values can be observed during the laser excitation in Fig. 4. These result from 
taking differences between single- and two-particle populations according to Eqs. (24)-(26), which both strongly oscillate along with the laser pulse on a short time scale.

We now turn to the results for the calculations on the short grid with CAP under the same condition of a focused laser and point out the differences. As shown in Fig. 3(a), after the pulse the $\left|L_{0}\right\rangle$-population remains at a constant minimum value of about 0.01 , even though ICD takes place as visible from the exponential decay of the resonance state. Through this, the main characteristic of the CAP becomes evident, namely the removal of continuum population. Thus, in the presence of a CAP continuum states generally give no contribution to the single-electron bound states Eqs. (24)-(26). The $\left|L_{0}\right\rangle$-population solely depends on the ground state population, which itself is completely depopulated by the end of the pulse. Anyhow, since the $\left|L_{0} C\right\rangle$-population is not immediately removed by the CAP, as a result of the time shift $t_{\text {fly }}\left(\mathrm{cf}\right.$. Sec. III A), $\left|L_{0}\right\rangle$ is always populated by the small amount mentioned above.

In Figs. 3(d) and 4(b) we find the results for the unfocused laser for calculations on the long grid without CAP. In comparison to the focused laser we observe here less population in $\left|L_{1} R_{0}\right\rangle$ and $\left|R_{0}\right\rangle$, while the $\left|L_{1}\right\rangle$-population is still similarly high. The reason is the increased impact of the direct ionization of the right QD that comes with the wide laser affecting now both QDs. Indeed, we find a local peak of the $\left|L_{0} C\right\rangle$-population during the pulse at about $7 \mathrm{ps}$ with an amount of 0.1 . This is a result of DI from the ground state $\left|L_{0} R_{0}\right\rangle$ into $\left|L_{0} C\right\rangle$ and further into $\left|L_{1} C\right\rangle$. The population of $\left|L_{1} C\right\rangle$ rises even higher, also via other excitation pathways, namely up to 0.3 until 11.5 ps and stays constant afterwards. This keeps the $\left|L_{1}\right\rangle$-population relatively high. In contrast, the probability to find either only the left or both QDs ionized is again very low (cf. $P_{\mathrm{CR}_{0}}$ and $P_{\mathrm{CC}}$ in Fig. $4(\mathrm{~b})$ ).

If we finally inspect the results for the calculations on the short grid with CAP under full laser exposition (cf. Fig. 3(b)) we find another irregularity caused by the CAP. Since the high contribution of $\left|L_{1} C\right\rangle$-population is removed by the CAP, the $L_{1}$-curve is forced to approach the resonance state curve. This also becomes clear from Eq. (25). Consequently, we observe a peak of $P_{\mathrm{L}_{1}}$ around 10 ps. This has been seen and discussed already in previous studies. $^{39}$

In the course of the study of the two differently sized grids, another inaccuracy caused by the CAP on the short grid concerning the resonance state population has become evident. According to Eqs. (25) and (26), the resonance state population should equal both, the $\left|L_{1}\right\rangle$ - 
and the $\left|R_{0}\right\rangle$-population after the ground state is completely depopulated. However, it is constantly smaller for both foci, cf. Figs. 3(a)+(b). It is also smaller than the resonance state population calculated on the long grid without CAP, cf. Figs. 3(e)+(f). We conclude that the combination of grid size and CAP position has not been chosen optimally for describing all aspects of the dynamics at equal accuracy. The CAP is too close to the QDs, causing a too strong overlap with the localized states. This we want to quantify by applying a CAP now to the long grid at $z= \pm 3250 \mathrm{~nm}$, thus with a distance from the QDs of factor 10 times the one for the small grid. As shown in Figs. 3(e)+(f), the resonance state dynamics on the long grid is now completely independent of the CAP, as the results are identical. In addition, we want to apply an alternative method for which the resonance state population on the short grid with CAP can be determined. By applying Eq. (29) we obtain an expression defined via the loss of the norm, namely $P_{L_{1} R_{0}}^{\mathrm{CAP}}(t)=\left|\Psi\left(t+t_{\mathrm{fly}}\right)\right|^{2}-P_{L_{0} R_{0}}^{\mathrm{CAP}}(t)$. It considerably improves on the long-time behavior of $P_{L_{1} R_{0}}$ making it similar to the one from the calculations on the long grid (see Figs. 3(e)+(f)). Despite these differences, we find the ICD rates from the long-term dynamics independent of grid size, usage of CAP and laser focus: $\Gamma_{\mathrm{ICD}, \text { foc. }}^{\mathrm{long}}=4.092 \times 10^{-3} \mathrm{meV}$, $\Gamma_{\text {ICD,foc. }}^{\text {long+CAP }}=4.086 \times 10^{-3} \mathrm{meV}, \Gamma_{\text {ICD,foc. }}^{\text {short+CAP }}=4.091 \times 10^{-3} \mathrm{meV}, \Gamma_{\text {ICD, unfoc. }}^{\text {long }}=4.092 \times$ $10^{-3} \mathrm{meV}, \Gamma_{\text {ICD, unfoc. }}^{\text {long+CAP }}=4.090 \times 10^{-3} \mathrm{meV}, \Gamma_{\text {ICD,unfoc. }}^{\text {short+CAP }}=4.091 \times 10^{-3} \mathrm{meV} . \quad$ Further-

more, the alternative method yields the same precision: $\Gamma_{\mathrm{ICD} \text {,foc. }}^{\text {short } \text {, alt }}=4.092 \times 10^{-3} \mathrm{meV}$, $\Gamma_{\mathrm{ICD}, \text { unfoc. }}^{\text {short }}=4.101 \times 10^{-3} \mathrm{meV}$.

To summarize the findings, the short- and long-term dynamics has been shown to be best represented for the calculations without CAP and the ICD process is prioritized by focusing the laser on the left QD only. Therefore, all our following calculations are performed under these conditions.

\section{B. Scan of the field strength}

The amount of population inversions between ground and resonance state can be controlled via the strength of the electric field Eq. (9). For the fixed pulse length $t_{\text {pulse }}$ the field strength $\eta_{n \pi}$ induces exactly $n \in \mathbb{N}$ inversions, or $n / 2$ Rabi oscillations. The respective pulse is referred to as $n \pi$-pulse.

The total ionization probability $\mathrm{P}_{\text {ion }}$ is a measure for the two ionization pathways, either the direct one or ICD. Here, it is calculated for a range of field strengths going from very 
low to high intensities. The scan of the field strength allows to determine the distinct $\eta_{n \pi}$ as shown below.

In our former studies, the same type of scan has been done for an unfocused laser on the short grid with CAP. ${ }^{40}$ Due to the CAP, we were able to propagate the wavefunction for long times $\left(t_{\mathrm{end}}=1278 \mathrm{ps}\right)$ and to deduce the ionization probability from the loss of the norm via $1-\left|\Psi\left(t_{\text {end }}\right)\right|^{2}$. The scan for the CAP-free calculations needs another measure for the ionization probability, because in this case the norm is unity at all times. Hence, we use the relation

$$
\mathrm{P}_{\text {ion }}=1-P_{L_{0} R_{0}}\left(t>t_{\text {pulse }}\right)
$$

instead. It is based on the fact that only those electrons do not contribute to the ionization probability which are left in the ground state after the laser pulse. Since the ground state population is constant for times larger than the pulse length (cf. Figs. 3(a)-(d)), the propagation can be terminated right after the pulse. Here, we used $t_{\text {end }}=19.2$ ps. As a consequence, this method is much faster than the previously applied one while giving the same results.

The scan for the CAP-free system is now performed under irradiation of a focused laser using both the space-resolved and the state-resolved method. As described in Sec. IIIB, for the state-resolved calculations the ionization rate $\Gamma_{\text {ion }}$ still needs to be determined. The parameter can be found through fitting the $\left|L_{1} R_{0}\right\rangle$-curve to the result from the space-resolved calculations. We found the field-independent rate $\Gamma_{\text {ion }}=10.230 \mathrm{meV}$ and the corresponding field-dependent rate defined by the field strength $\Gamma_{\text {ion }, \eta_{\pi}}=\left(\eta_{\pi} / 2\right)^{2} \times \Gamma_{\text {ion }}=2.256 \times 10^{-3} \mathrm{meV}$.

Fig. 5 shows the ionization probability $\mathrm{P}_{\text {ion }}$ with respect to the field strength given in units of $\eta_{\pi}$ for the space-resolved (solid black) and state-resolved calculations (dashed black). The pulse duration is kept constant at $t_{\text {pulse }}=14.1 \mathrm{ps}$ as has been used before for the $\pi$-pulse calculations. The gray curve indicates the relative difference $\Delta$ between both methods. An oscillatory behavior of the ionization probability can be observed for both methods. The maxima being all $\mathrm{P}_{\text {ion }}=1$, represent zero population in the ground state at the pulse end. A minimum of the ionization probability is reached when the population within the QDs is inverted back to the ground state at the end of the pulse, such that ICD is disabled. Based on these explanations we conclude that the oscillations of the ionization probability follow the Rabi cycles between ground and resonance state, hence maxima are found at field 


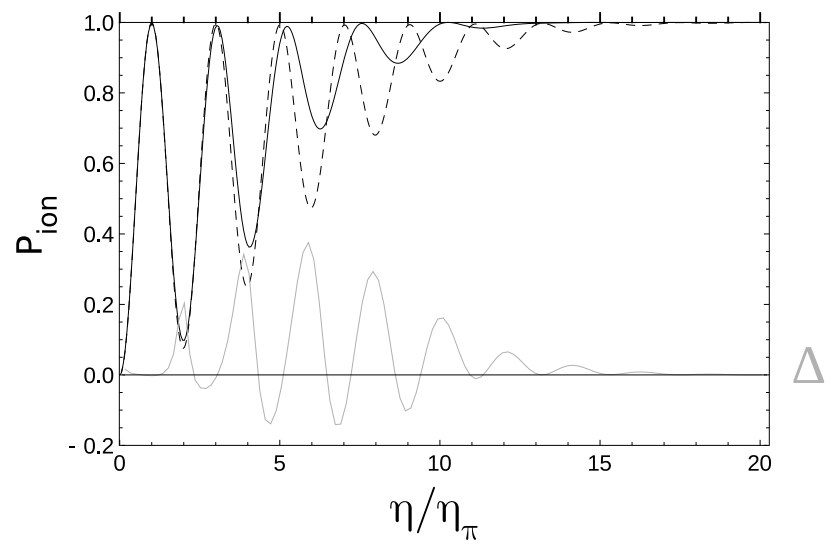

FIG. 5. Ionization probability $\mathrm{P}_{\text {ion }}$ for the scan over the field strength $\eta$ given in units of $\eta_{\pi}$ for a constant pulse length $t_{\text {pulse }}=14.1 \mathrm{ps}$ and resonant excitation energy $\omega_{\mathrm{RES}}=5.179 \mathrm{meV}$. Calculations for the CAP-free system under irradiation of a focused laser pulse on the left QD are compared for the space-resolved (solid black) and state-resolved (dashed black) method. The gray curve $\Delta$ shows the relative difference of the two methods with respect to the space-resolved results.

strengths for odd $n \pi$-pulses and minima at even $n \pi$-pulses. Contrary to the maxima, the minima have no constant value of $\mathrm{P}_{\text {ion }}$, but increase with higher field strengths until finally fading into a constant value of one. This is due to the fact that the probability for ionizing the left QD increases with growing field strength. Indeed, for the $\pi$-pulse we only find $1 \%$ of all population in $\left|C R_{0}\right\rangle$ after the pulse has finished, cf. Fig. 6. For the field strength that induces a $5 \pi$-pulse it is already $51 \%$, and $95 \%$ for the $10 \pi$-pulse. This in consequence means, the impact of ICD becomes negligible for growing laser intensities.

In our former studies for an unfocused laser only three population inversions had been visible, as the impact of direct ionization was strong already for low field strengths allowing for massive ionization of the right QD. ${ }^{40}$ Now, for the focused laser the scan shows much more inversions. Since direct ionization of the right electron is minimized, the system is able to return to its ground state still for highest field strengths. In total we see 12 population inversions for the space-resolved calculations and 19 for the state-resolved method before the population in the QDs becomes less than $0.05 \%$.

Let us now turn to the differences of both methods. For a focused laser the state-resolved method basically describes single-photon processes only. Thus, if multi-photon excitations are negligible, both methods should give equal results. This is indeed the case for the field 


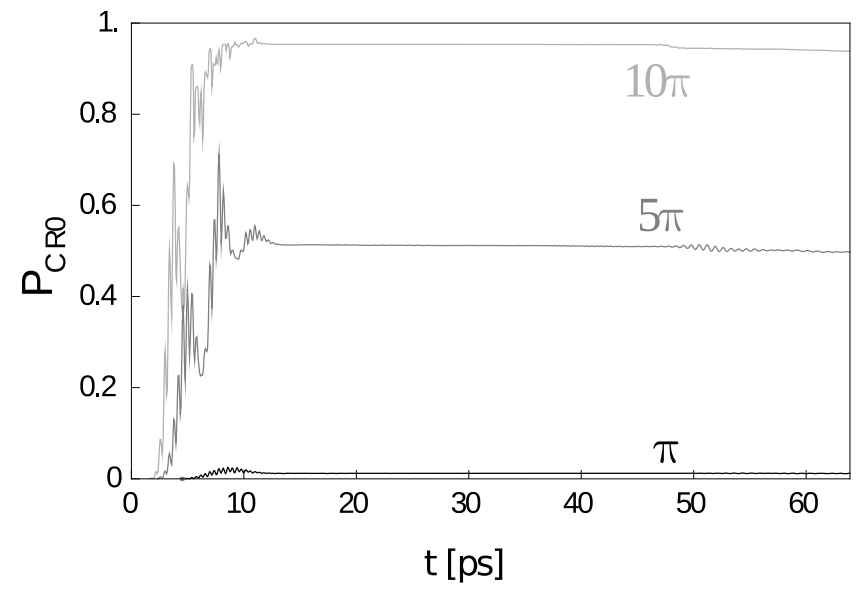

FIG. 6. Population of $\left|C R_{0}\right\rangle$ with respect to time for a $\pi$-, $5 \pi$ - and $10 \pi$-pulse with focus on the left QD.

strengths range up to the first population inversion $\left(\eta \leq \eta_{\pi}\right)$ in agreement with our previous findings on the $\pi$-pulse excitations in Sec. IV A. As higher excited states are excluded in the state-resolved ansatz, a growing discrepancy between the two methods can be observed from the relative difference curve $\Delta$. It gains amplitude with increasing field strength and attenuates where the ionization probabilities obtained with both methods converge towards unity.

The distance between extrema increases with growing $n$ as can be clearly seen for the space-resolved calculations, cf. Table I and Fig. 7. With increasing field strength the resonance condition for the system changes as the electronic levels are shifted when being exposed to the external field. However, we use the same excitation energy $\omega_{\mathrm{RES}}=5.179 \mathrm{meV}$ as it was initially determined without any field interactions throughout the whole scan. The extrema are further found in groups of two, i.e. one pair for each Rabi cycle, which all have in common of being approximately parallel to the linear function $n$ (Fig. 7).

We applied the $n \pi$-pulses as determined in the scan and listed in Table I. In Fig. 8 the resonance state populations (solid lines) are shown (a) for $n=1,3,5$ and (b) for $n=2,4,6$. For a better resolution of the initial dynamics the process is depicted only up to 30 ps. We find that after each Rabi-cycle an additional maximum adds to the resonance state population. The first maximum is decreasing and appearing earlier in time for increasing $n$. The effect originates from the rising impact of DI of the left QD for increasing field strengths, as discussed in Sec. IV B. For the even-numbered $n \pi$-pulses, the resonance state 
TABLE I. Field strengths $\eta_{n \pi}$ of the $n \pi$-pulses in units of $\eta_{\pi}=2.82 \times 10^{4} \mathrm{Vm}^{-1}$ as obtained from the scan for the space-resolved calculations with $t_{\text {pulse }}=14.1 \mathrm{ps}$ and a focused laser. $\Gamma_{\mathrm{ICD}}$ is determined from the long-term exponential decay of the resonance state for calculations on the long grid with CAP.

\begin{tabular}{|r|r|r|c|}
\hline$n$ & $\eta_{n \pi} / \eta_{\pi}$ & $\mathrm{I} / \mathrm{Wcm}^{-2}$ & $\Gamma_{\mathrm{ICD}} / \mathrm{meV}$ \\
\hline 1 & 1.00 & 1368 & $4.078 \times 10^{-3}$ \\
\hline 2 & 1.99 & 5433 & - \\
\hline 3 & 3.05 & 12723 & $4.088 \times 10^{-3}$ \\
\hline 4 & 4.07 & 22588 & - \\
\hline 5 & 5.24 & 37479 & $4.090 \times 10^{-3}$ \\
\hline 6 & 6.28 & 53747 & - \\
\hline 7 & 7.62 & 79319 & $4.074 \times 10^{-3}$ \\
\hline 8 & 8.73 & 104100 & - \\
\hline 9 & 10.27 & 143939 & $4.076 \times 10^{-3}$ \\
\hline 10 & 11.36 & 176118 & - \\
\hline 11 & 14.86 & 301412 & $4.281 \times 10^{-3}$ \\
\hline 12 & 15.90 & 345084 & - \\
\hline
\end{tabular}

is completely depopulated by the end of the pulse (cf. Fig. 8(b)) thus preventing further ICD. For an odd number of inversions the population in the dots is predominantly in the resonance state by the end of the pulse followed by the exponential decay via ICD (cf. Fig. 8(a)). The decay rates for up to the $11 \pi$-pulse are determined from such type of figures, cf. Table I. However, for this purpose we performed extra calculations on the long grid now with CAP (not shown here). This was to reach a long final propagation time of 320 ps while eliminating backscattering, cf. Sec IV A. The decay rates determined for all odd $n$ are in good agreement with each other. Only for the $11 \pi$-pulse a slight inconsistency can be found. The reason for this is purely numerical. The decay starts from a population of about 0.001 being left in the resonance state, hence making the fit extremely sensitive to errors. Compared to the previously found result in Sec. IV A a slightly different $\Gamma_{\text {ICD }}$ is found for $n=1$, since before the decay rates were determined from propagations until only 60 ps.

Finally, a comparison to a state-resolved calculation is made for $n=3$ (dashed line), 


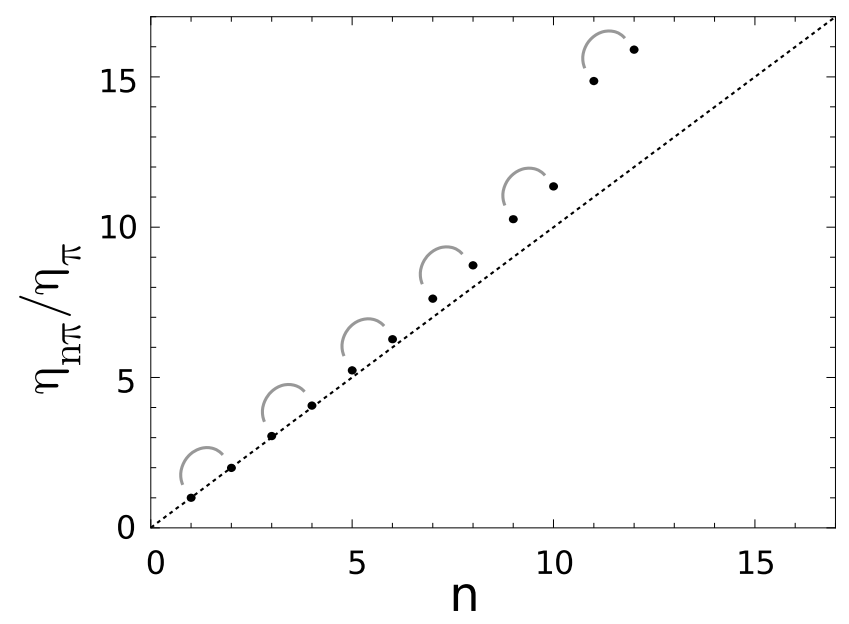

FIG. 7. The $n \pi$-pulse field strengths $\eta_{n \pi}$ in units of $\eta_{\pi}$ vs. the number of inversions $n$ as obtained from the space-resolved calculations under application of the focused laser, cf. Table I. The dashed line is equal to the linear function $n$. Pairs of $\eta_{n \pi} / \eta_{\pi}$ ratios are indicated by bent lines corresponding to one completed Rabi cycle.

showing slight deviations between both methods. This confirms our expectations that the results for the two methods start to differ when going beyond the $\pi$-pulse as the scan (Sec. IV B) already showed.

\section{CONCLUSION}

The dynamics of two electrons in a pair of quantum dots has been studied with view on the strength and focus of the exciting laser pulse that determines the range of excited states and is a crucial factor for the two competing ionization pathways, a direct one and the inter-Coulombic decay.

When the laser is focused on the left QD ionization takes place mostly via ICD, whereas upon full penetration direct ionization of the right $\mathrm{QD}$ is of significance. In extension to former studies, new insights are given on multi-photon effects that become the more relevant the stronger and less focused the pulse is.

Such detailed analysis was now possible for two reasons. Due to the focused laser we were able to resolve in a scan over the field strengths six rather than only two Rabi cycles in the non-focused case. Moreover, the utilization of a long calculation grid without absorbing boundaries facilitated the development of extended analysis methods which made it possible 

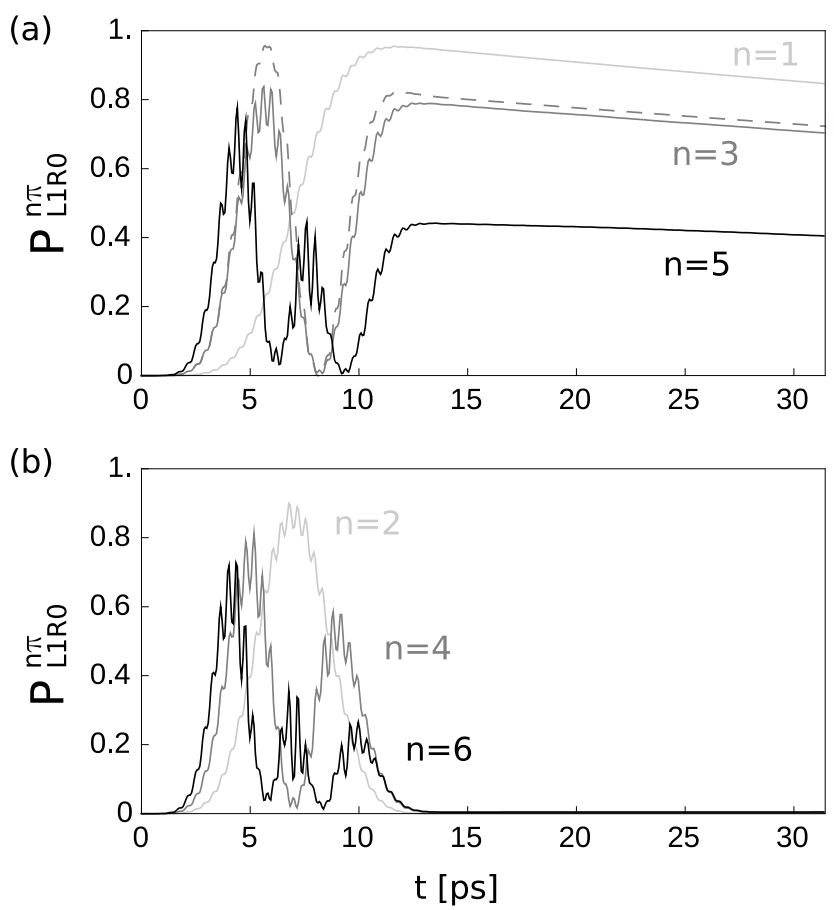

FIG. 8. Resonance state populations for the $n \pi$-laser pulse excitations for (a) $n=1,3,5$ and (b) $n=2,4,6$ with a focus on the left $\mathrm{QD}$. The space-resolved calculations are performed on the long CAP-free grid (solid lines). A comparison is made to the state-resolved method for the $3 \pi$-pulse (dashed line). For the constant pulse length $t_{\text {pulse }}=14.1 \mathrm{ps}$ the field strengths $\eta_{n \pi}$ are applied as obtained from the scan (cf. Sec. IV B and Table I).

to refine our understanding on the populations of the different continuum states in addition to that of the bound and resonance states.

In passing we investigated the influence of complex absorbing potentials and grid sizes. CAPs on small grids, which will be of utter importance for future calculations with additional continuum dimensions, were found to disturb the accurate determination of relative state populations, but still to render exact decay widths for ICD, the quantity we are ultimately interested in.

\section{ACKNOWLEDGMENTS}

First of all, we would like to acknowledge the people who made this work possible by providing their code for state-resolved calculations (Ying-Chih Chiang) and preliminary calculations with the focused laser (Maximilian Menger). Further we thank the Deutsche 
Forschungsgemeinschaft (BA 3770/2-1) and the Volkswagen Foundation (Freigeist Fellowship 98525) for funding.

\section{REFERENCES}

${ }^{1}$ M. A. Reed, J. N. Randall, R. J. Aggarwal, R. J. Matyi, T. M. Moore, and A. E. Wetsel, Phys. Rev. Lett. 60, 535 (1988).

${ }^{2}$ M. A. Kastner, Phys. Today 46, 24 (1993).

${ }^{3}$ L. P. Kouwenhoven, T. H. Oosterkamp, M. W. Danoesastro, M. Eto, D. G. Austing, T. Honda, and S. Tarucha, Science 278, 1788 (1997).

${ }^{4}$ P. Alivisatos, Pure Appl. Chem. 72, 3 (2000).

${ }^{5}$ A. Zrenner, J. Chem. Phys. 112, 7790 (2000).

${ }^{6}$ P. M. Petroff, A. Lorke, and A. Imamoglu, Phys. Today 54, 46 (2001).

${ }^{7}$ W. G. van der Wiel, S. De Franceschi, J. M. Elzerman, T. Fujisawa, S. Tarucha, and L. P. Kouwenhoven, Rev. Mod. Phys. 75, 1 (2002).

${ }^{8}$ A. Sauerwald, T. Kümmell, G. Bacher, A. Somers, R. Schwertberger, J. P. Reithmaier, and A. Forchel, Appl. Phys. Lett. 86, 253112 (2005).

${ }^{9}$ J. H. Wei and K. S. Chan, J. Appl. Phys. 97, 123524 (2005).

${ }^{10}$ Y. Yin and A. P. Alivisatos, Nature 437, 664 (2005).

${ }^{11}$ L. Wang, A. Rastelli, S. Kiravittaya, M. Benyoucef, and O. G. Schmidt, Adv. Mater. 21, 2601 (2009).

${ }^{12}$ J. Salfi, S. Roddaro, D. Ercolani, L. Sorba, I. Savelyev, M. Blumin, H. E. Ruda, and F. Beltram, Semicond. Sci. Technol. 25, 024007 (2010).

${ }^{13}$ O. Benson, C. Santori, M. Pelton, and Y. Yamamoto, Phys. Rev. Lett. 84, 2513 (2000).

${ }^{14}$ P. Michler, A. Kiraz, C. Becher, W. V. Schoenfeld, P. M. Petroff, L. Zhang, E. Hu, and A. Imamoglu, Science 290, 2282 (2000).

${ }^{15}$ D. Press, S. Götzinger, S. Reitzenstein, C. Hofmann, A. Löffler, M. Kamp, A. Forchel, and Y. Yamamoto, Phys. Rev. Lett. 98, 117402 (2007).

${ }^{16}$ M. Arcari, I. Söllner, A. Javadi, S. Lindskov Hansen, S. Mahmoodian, J. Liu, H. Thyrrestrup, E. H. Lee, J. D. Song, S. Stobbe, and P. Lodahl, Phys. Rev. Lett. 113, 093603 (2014).

${ }^{17}$ M. G. Bawendi, P. J. Carroll, W. L. Wilson, and L. E. Brus, J. Chem. Phys. 96, 946 
(1992).

${ }^{18}$ H. Drexler, D. Leonard, W. Hansen, J. P. Kotthaus, and P. M. Petroff, Phys. Rev. Lett. 73,2252 (1994).

${ }^{19}$ S. Sauvage, P. Boucaud, F. H. Julien, J.-M. Gérard, and V. Thierry-Mieg, Appl. Phys. Lett. 71, 2785 (1997).

${ }^{20}$ P. Guyot-Sionnest and M. A. Hines, Appl. Phys. Lett. 72, 686 (1998).

${ }^{21}$ S. Maimon, E. Finkman, G. Bahir, S. E. Schacham, J. M. Garcia, and P. M. Petroff, Appl. Phys. Lett. 73, 2003 (1998).

${ }^{22}$ S.-F. Tang, S.-Y. Lin, and S.-C. Lee, Appl. Phys. Lett. 78, 2428 (2001).

${ }^{23}$ I. Gur, N. A. Fromer, M. L. Geier, and A. P. Alivisatos, Science 310, 462 (2005).

${ }^{24}$ A. J. Nozik, M. C. Beard, J. M. Luther, M. Law, R. J. Ellingson, and J. C. Johnson, Chem. Rev. 110, 6873 (2010).

${ }^{25}$ F. Meinardi, H. McDaniel, F. Carulli, A. Colombo, K. A. Velizhanin, N. S. Makarov, R. Simonutti, V. I. Klimov, and S. Brovelli, Nat. Nanotechnol. 10, 878 (2015).

${ }^{26}$ I. I. Rabi, Phys. Rev. 51, 652 (1937).

${ }^{27}$ I. I. Rabi, S. Millman, P. Kusch, and J. R. Zacharias, Phys. Rev. 55, 526 (1939).

${ }^{28}$ H. C. Torrey, Phys. Rev. 76, 1059 (1949).

${ }^{29}$ G. B. Hocker and C. L. Tang, Phys. Rev. Lett. 21, 591 (1968).

${ }^{30}$ R. Binder, S. W. Koch, M. Lindberg, N. Peyghambarian, and W. Schäfer, Phys. Rev. Lett. 65, 899 (1990).

${ }^{31}$ W. Schäfer and K. Henneberger, Phys. Status Solidi B 159, 59 (1990).

${ }^{32}$ S. T. Cundiff, A. Knorr, J. Feldmann, S. W. Koch, E. O. Göbel, and H. Nickel, Phys. Rev. Lett. 73, 1178 (1994).

${ }^{33}$ L. C. Andreani, G. Panzarini, and J.-M. Gérard, Phys. Rev. B 60, 13276 (1999).

${ }^{34}$ A. Schülzgen, R. Binder, M. E. Donovan, M. Lindberg, K. Wundke, H. M. Gibbs, G. Khitrova, and N. Peyghambarian, Phys. Rev. Lett. 82, 2346 (1999).

${ }^{35}$ H. Kamada, H. Gotoh, J. Temmyo, T. Takagahara, and H. Ando, Phys. Rev. Lett. 87, 246401 (2001).

${ }^{36}$ T. H. Stievater, X. Li, D. G. Steel, D. Gammon, D. S. Katzer, D. Park, C. Piermarocchi, and L. J. Sham, Phys. Rev. Lett. 87, 133603 (2001).

${ }^{37}$ G. Y. Slepyan, A. Magyarov, S. A. Maksimenko, A. Hoffmann, and D. Bimberg, Phys. Rev. B 70, 045320 (2004). 
${ }^{38}$ J. Luo, W. Lai, D. Lu, C. Du, Y. Liu, S. Gong, D. Shi, and C. Guo, J. Phys. B: At. Mol. Opt. Phys. 45, 035402 (2012).

${ }^{39}$ A. Bande, J. Chem. Phys. 138, 214104 (2013).

${ }^{40}$ A. Haller, Y.-C. Chiang, M. Menger, E. F. Aziz, and A. Bande, Chem. Phys. 482, 135 (2017).

${ }^{41}$ L. S. Cederbaum, J. Zobeley, and F. Tarantelli, Phys. Rev. Lett. 79, 4778 (1997).

${ }^{42}$ I. Cherkes and N. Moiseyev, Phys. Rev. B 83, 113303 (2011).

${ }^{43}$ A. Bande, K. Gokhberg, and L. S. Cederbaum, J. Chem. Phys. 135, 144112 (2011).

${ }^{44}$ F. Grossmann, Theoretical Femtosecond Physics (Springer-Verlag, Berlin Heidelberg, 2008).

${ }^{45}$ L. V. Keldysh, Sov. Phys. JETP-USSR 20, 1307 (1965).

${ }^{46}$ F. A. Ilkov, J. E. Decker, and S. L. Chin, J. Phys. B: At. Mol. Opt. Phys. 25, 4005 (1992).

${ }^{47}$ H.-D. Meyer, U. Manthe, and L. S. Cederbaum, Chem. Phys. Lett. 165, 73 (1990).

${ }^{48}$ U. Manthe, H.-D. Meyer, and L. S. Cederbaum, J. Chem. Phys. 97, 3199 (1992).

${ }^{49}$ M. Beck, Phys. Rep. 324, 1 (2000).

${ }^{50}$ H.-D. Meyer, F. Gatti, and G. A. E. Worth, Multidimensional Quantum Dynamics MCTDH Theory and Applications (Wiley-VCH, Weinheim, 2009).

${ }^{51}$ R. Kosloff and D. Kosloff, J. Comput. Phys. 63, 363 (1986).

${ }^{52}$ U. V. Riss and H.-D. Meyer, J. Phys. B: At. Mol. Opt. Phys. 26, 4503 (1993).

${ }^{53}$ U. V. Riss and H.-D. Meyer, J. Chem. Phys. 105, 1409 (1996).

${ }^{54}$ S. Selst $\varnothing$ and S. Kvaal, J. Phys. B: At. Mol. Opt. Phys. 43, 065004 (2010).

${ }^{55}$ S. Kvaal, Phys. Rev. A 84, 022512 (2011).

${ }^{56}$ F. M. Pont, A. Bande, and L. S. Cederbaum, J. Phys. Condens. Matter 28, 241304 (2016).

${ }^{57}$ P. Dolbundalchok, D. Peláez, E. F. Aziz, and A. Bande, J. Comput. Chem. 37, 2249 (2016).

58 "Electronic archive: New semiconductor materials. characteristics and properties," http://www.ioffe.ru/SVA/NSM/Semicond/GaAs/basic.html, Accessed: 2018-05-01.

${ }^{59}$ S. Bednarek, B. Szafran, T. Chwiej, and J. Adamowski, Phys. Rev. B 68, 045328 (2003).

${ }^{60}$ A. Bande, F. M. Pont, P. Dolbundalchok, K. Gokhberg, and L. S. Cederbaum, EPJ Web Conf. 41, 04031 (2013).

${ }^{61}$ D. J. Tannor, Introduction to Quantum Mechanics (University Science Books, USA, 2007).

${ }^{62}$ A. Jäckle and H.-D. Meyer, J. Chem. Phys. 104, 7974 (1996). 
${ }^{63}$ A. Jäckle and H.-D. Meyer, J. Chem. Phys. 109, 3772 (1998).

${ }^{64}$ J. C. Light and T. Carrington, "Discrete variable representations and their utilization," in Advances in Chemical Physics (Wiley-Blackwell, 2007) pp. 263-310.

${ }^{65}$ Y.-C. Chiang, P. V. Demekhin, A. I. Kuleff, S. Scheit, and L. S. Cederbaum, Phys. Rev. A 81, 032511 (2010).

${ }^{66}$ P. V. Demekhin and L. S. Cederbaum, Phys. Rev. A 83, 023422 (2011). 